\title{
Manangement of Endotracheal Intubation in Suspected or Confirmed COVID-19 Patients
}

\author{
Nazlihan BOYACI DUNDAR ${ }^{1}$
}

${ }^{1}$ Gazi University School of Medicine Medical Intensive Care Unit, Ankara, Turkey

Cite this article as: Boyaci Dundar N. Manangement of Endotracheal Intubation in Suspected or Confirmed Coronavirus Disease 2019 (COVID-19) Patients. J Crit Intensive Care 2020; 11(Suppl. 1):10-11.

Corresponding Author: Nazlihan Boyaci Dundar

E mail: nazlihan_boyaci@yahoo.com

CCopyright 2020 by Turkish Society of Medical and Surgical Intensive Care Medicine - Available online at www.jcritintensivecare.org

Received: May 22, 2020 Accepted: May 27, 2020 Available online: Jun 22, 2020

\begin{abstract}
Severe acute respiratory syndrome coronavirus 2 (SARS-CoV-2) has been described as a novel coronavirus causing a pandemic of the novel corona virus disease 2019 (COVID-19). It is highly contagious and the main transmission way is respiratory droplets and close contact with contaminated surfaces. The aerosol generating procedures caries additional risk for healthcare workers. Since endotracheal intubation is a vulnerable procedure for aerosol generation, the airway manangement of suspected or confirmed COVID-19 patients is high risk to staff and patient In this review we aimed to highlight the important points for the safe and effective endotracheal intubation procedure for suspected or confirmed COVID-19 patients.
\end{abstract}

Keywords: COVID-19, Airway Manangement, Endotracheal Intubation, Planning and Preperation

\section{Introduction}

Severe acute respiratory syndrome coronavirus 2 (SARS-CoV-2) has been described as a novel coronavirus causing a pandemic of novel corona virus disease 2019 that is recently announced by the World Health Organization (WHO)(1). It is highly contagious and the main transmission way is respiratory droplets and close contact with contaminated surfaces $(2,3)$. Since endotracheal intubation is a vulnerable procedure to aerosol generation, the airway manangement of suspected or confirmed COVID-19 patients is high risk to staff and patient $(4,5)$. To prevent contamination of healthcare workers and perform this procedure safely, proper planning and preparation is crucial. The recommendations with the following headings have been adopted from the current guidelines and literature for the purpose of maintaining staff safety while providing timely and effective airway management.

\section{Institutional Planning and Preparation}

Institutional preparation is vital to optimize staff and patient safety in airway management of COVID-19 patient Appropriate planning and preparation should be done about the team, enviroment and equipments according to the available resources of the health institution. We can summarize the important points to be considered in planning under some headings.

- A detailed plan has to be documentated according to the resources of the health institution. The validity and applicability of this plan should also be assured.

- Negative pressure ventilation rooms with an ante-room are ideal to minimise exposure to aerosol and droplet particles, if not possible, choose a normal pressure room with strict door policy $(4,5)$.

- If possible the intubation teams focused on this issue should be created. This may improve familiarity, compliance and efficiency of airway manangement plan and also provide to use existing resources effectively (5).

- For minimizing the staff; experienced specialist doctor (operator), assistant doctor or health technician assisting airway application and nurse being present during the procedure $(4,5)$.

- Define the roles clearly and use a clear communication language (5). It can be difficult to hear through personal protective equipment (PPE). Clear, simple, concise language should be used and voices need to be raised to be heard through PPE (5). 
- The major challenge in the airway manangement of these population is limited physiological reserves due to the acute respiratory distress syndorme (ARDS). The emergency intubation during severe hypoxia or cardiac/respiratory arrest is an additional risk for healtcare workers (5). To minimize the risk, it has to be considered early intubation to prevent be unpreparedness in emergency (5). Also, meticulous assessment of the airway to predict possible difficult intubation is very important for this respect.

\section{Recommendations Before The Process of Airway Manangement}

The final checks before the procedure are very important for the safe and effective airway manangement. Routine use of an intubation checklist and kit dump, preferably specifically modified for the COVID-19 patient group, is recommended $(4,5)$. The important topics to be considered in this regard are;

- Ensure the hand hygiene and wear the full PPE (2). A spotter, observes that all staff have donning and doffing of PPE correctly, may help protect task-focused staff from PPE breaches (5). If not possible, institutional training and practicing on the correct use of personal protective equipment should be provided at frequent intervals.

- Check the intubation trolley and prepare the medicines (sedative agents and neuromuscular blokan) in advance. It is always prefered disposable item over reusable equipment (5). If not possible, make sure that these equpments are sterilized under appropriate conditions.

- Ensure the presence of working aspirator system, monitor, waveform etCO2 system and ventilator (closed position) (4).

- Before the procedure place the bacteria/viral (HME) filters in places such as the expiratory line of ventilator, breathing circuit and ambu mask.

- Use closed-loop suction to minimize aerosol generation $(4,5)$.

- Ensure a working, checked intravenous access is present (4).

- Provide a vasopressor for bolus or infusion for managing hypotension (4).

\section{Recommendations For Endotracheal Intubation}

It is crucial to minimise the risk of aerosol generation during endotracheal intubation (5). For this purpose rapid sequential intubation should be performed by the most experienced staff using video laryngoscope to minimize droplet contamination (4). It is also important for the first attempt success (4). The preoxygenation period is another condition that requires attention for preventing droplet spread. The important topics to be considered in this regard are;

- Provide oxygen support with the nasal cannula, simple oxygen mask or non-rebreather mask at the lowest possible flow to ensure oxygenation.

- Avoide preoxygenation with ambu-mask for aerolization. If necessary, a viral filter sholud be placed between the ambu and the mask, and the mask should be fully seated on the face with two hands to minimize air leakage $(4,5)$.
- Before the attempt, ensure the neuromuscular blockade to prevent coughing.

- If avaliable, use videolaryngoscopy with a seperate screen. This enables the operator to stay further the airway (4).

- After endotracheal intubation, inflate the tracheal tube cuff to seal the airway before starting ventilation (4).

- Confirm tracheal intubation with waveform etCO2 if possible, since the stethoscope may cause disruption in the integrity of the PPE (4).

- Cuff pressure should be monitored with a cuff manometer to ensure an adequate seal $(4,5)$.

- Avoid the processes that will disrupt circuit integrity.

- If necessary, use "aerochamber" instead of nebulizer for inhaler treatment.

- A nasogastric tube should be placed at the time of intubation to avoid further close contact with the airway $(4,5)$

\section{Conclusion}

SARS-CoV-2 that causes COVID-19 is a highly contagious virus. The aerosol generating procedures such as endotracheal intubation, caries additional risk for healthcare workers. To prevent contamination of healthcare workers and perform this procedure safely, proper planning and preparation is crucial. The institutional preparation should be done according to the available local resources. In this context, it is recommended to document a detailed plan and routine use of an intubation checklist and kit dump. To minimise the risk of aerosol generation during endotracheal intubation, rapid sequential intubation should be performed by the most experienced staff via video laryngoscope.

\section{References}

1. World Health Organization. WHO characterizes COVID-19 as a pandemic-11 March 2020. https://www.who.int/emergencies/ diseases/novelcoronavirus-2019/events-as-they-happen.

2. World Health Organization, Infection prevention and control during health care when novel coronavirus ( $\mathrm{nCoV}$ ) infection is suspected Interim guidance. January 2020. https://www.who.int/emergencies/ diseases/novel-coronavirus-2019/technical-guidance/health-workers

3. Wu Z, McGoogan JM. Characteristics of and Important Lessons From the Coronavirus Disease 2019 (COVID-19) Outbreak in China: Summary of a Report of 72314 Cases From the Chinese Center for Disease Control and Prevention. JAMA. 2020.

4. Cook T. M, El-Boghdadly K, McGuire B, et al. Consensus guidelines for managing the airway in patients with COVID-19: Guidelines from the Difficult Airway Society, the Association of Anaesthetists the Intensive Care Society, the Faculty of Intensive Care Medicine and the Royal College of Anaesthetists. Anaesthesia. 2020 Jun;75(6): 785-799.

5. Bewster DJ, Chrimes N, Do TB, et al. Consensus Statement: Safe Airway Society Principles of Airway Management and Tracheal Intubation Specific to the COVID-19 Adult Patient Group. Med J Aust 2020 May 1 